\title{
Transient dilation of the left ventricular cavity observed during myocardial perfusion imaging: What is its incremental diagnostic value
}

\author{
Raymond Taillefer, MD, FRCP, ABNM ${ }^{a, b}$ \\ a Department of Nuclear Medicine, CISSS Montérégie-Centre, l'Hôpital du Haut-Richelieu, \\ St-Jean-sur-Richelieu, Canada \\ b Department of Radiology, Radio-oncology and Nuclear Medicine, Université de Montréal, \\ Montreal, Canada
}

Received Sep 12, 2016; accepted Sep 12, 2016

doi:10.1007/s12350-016-0701-2

\section{See related article, pp. 586-592}

Transient ischemic dilation (TID) of the left ventricle (LV), defined as the apparent significant enlargement in the LV size on post-stress images in comparison to the rest images on SPECT radionuclide myocardial perfusion imaging (MPI), has been recognized as an important marker of severe and extensive coronary artery disease (CAD) and indicative of high risk of cardiac events as well, even when MPI studies appear to be normal. ${ }^{1-10}$ TID is predictive of proximal left anterior descending arteries or triple vessels with greater than $90 \%$ stenosis. This has been shown with various MPI protocols and radionuclides, with both treadmill stress test and vasodilators (dipyridamole and adenosine). The frequency of TID on radionuclide MPI has been reported to range from $3 \%$ to $37 \%^{8,9,11,12}$ depending on several factors such as the type of patients population (with or without previously known CAD, previous myocardial infarction, advanced age, diabetes mellitus, LV hypertrophy, elevated LV end-diastolic pressure, etc.), the gender (difference between male and female patients), the method used to define TID (visual analysis versus the use of automatic quantitative assessment of TID), the quantitative upper limits to define TID, the stress modalities (exercise vs

Reprint requests: Raymond Taillefer, MD, FRCP, ABNM, Department of Nuclear Medicine, CISSS Montérégie-Centre, l'Hôpital du HautRichelieu, 920 boul du séminaire nord, St-Jean-sur-Richelieu, Canada; rtaillefer@hotmail.com

J Nucl Cardiol 2018;25:593-5.

$1071-3581 / \$ 34.00$

Copyright (c) 2016 American Society of Nuclear Cardiology. pharmacologic stress MPI), the radioisotopes used ( ${ }^{201}$ thallium vs $99 \mathrm{mTc}$-labeled MPI agents), and the imaging protocols (single radiotracers, dual radiotracers ${ }^{201}$ T1-99mTc-MPI, same-day rest-stress, same-day stress-rest imaging sequences, 2-day protocols, etc.).

The exact underlying pathophysiologic mechanisms of TID remain unclear. The involved mechanisms are likely to be varied, either related to pathologic or physiologic changes. The TID ratio measured from SPECT MPI may reflect true stress-induced stunning of the LV, extensive subendocardial ischemia, or a combination of the two mechanisms. There is still no definite conclusion about the underlying mechanisms of TID. The presence of severe stress-induced diffuse subendocardial hypoperfusion resulting in the non-visualization of extensive amount of the subendocardial myocardium (producing a visually larger LV cavity on SPECT images), following stress in comparison to the rest normal study, is probably the most common pathologic mechanism causing an apparent TID (or " "pseudo-dilation,", as opposed to true dilation), ${ }^{1,8,13}$ although some studies have shown data supporting the concept that TID represented true LV dilation. ${ }^{14-16}$ In 1987 , Weiss et al ${ }^{16}$ postulated that the finding of TID was likely to represent extensive myocardial ischemia because it is reversible; the amount of ischemia has to be significant enough to cause sometimes true transient ventricular enlargement as detected on planar ${ }^{201}$ thallium studies; and the ischemia is likely to be quite severe because it lasts for at least 30 minutes after the stress, a time at which "usual" stress-induced myocardial ischemia would be expected to have been resolved. They found that LV volumes calculated from the epicardial borders were also enlarged, indicating that if TID was due to diffuse subendocardial ischemia alone, it would have been expected that TID would be observed with the use of 
endocardial but not epicardial boundaries for the TID ratio calculation. Using gated ${ }^{201}$ thallium dipyridamole stress and redistribution MPI, Hung et $\mathrm{al}^{14}$ found a greater enlargement of post-stress end-systolic volume than of end-diastolic volume in the group of patients with TID compared to those without TID, suggesting that there should be explanations other than apparent LV dilation to explain the occurrence of TID, since subendocardial hypoperfusion in patients with TID should give the same degree of enlargement for both end-diastolic and end-systolic volumes. Other studies with $99 \mathrm{mTc}$-tetrofosmin ${ }^{17}$ showed that patients with ischemic myocardial stunning had a significant increase in end-systolic volume post-stress but not in end-diastolic volumes. These findings suggest that TID represents true ventricular dilation rather than just an apparent LV dilation, related to myocardial ischemic stunning manifested by stress-induced decrease of the LV ejection fraction and worsening of wall motion. This finding has been described on both exercise and pharmacologic MPI stress tests. ${ }^{18,19}$ It is also very likely that if SPECT MPI is performed very early after the radiotracer injection at stress, the likelihood that post-stress myocardial stunning and TID will be observed is higher. No matter the exact mechanism of TID, both true dilation and extensive subendocardial ischemia play a significant role in TID, and both are caused by extensive stress-induced abnormality. Microvascular disease and/ or impaired coronary flow reserve have also been postulated as potential mechanisms. ${ }^{20}$

Although TID is not always necessarily associated with obstructive CAD as it has been described in patients with diabetes mellitus, LV hypertrophy, and patients with a hypertensive blood pressure response to exercise, ${ }^{20,21}$ it is widely accepted that the principal added value of TID in MPI is that it is a marker of severe and extensive CAD. This parameter is also an independent adverse prognostic predictor. Patients with an entirely normal exercise or vasodilator MPI study but with TID have been shown to have a significantly increase in follow-up cardiac events in comparison to patients with no TID and normal MPI study. Implementation of non-perfusion imaging parameters such as increased radiotracer lung uptake on post-stress images or TID also improves the diagnostic accuracy of MPI. In this regard, this issue of the Journal of Nuclear Cardiology includes an interesting study by Gultekin et $\mathrm{al}^{22}$ on the value of TID for detecting restenosis after coronary artery revascularization using either a same-day or a 2-day 99mTc-sestamibi SPECT MPI protocol in 104 patients who had recent coronary revascularization and recurrent symptoms with a repeat coronary angiography. Both treadmill exercise and dipyridamole vasodilator stress were used. The sensitivity of SPECT MPI alone for detecting significant obstructive disease was $61 \%$ while this number significantly increased to $93 \%$ $(P<.0001)$ by adding TID as a diagnostic criterion to MPI. The relatively low sensitivity of $61 \%$ of MPI defects for detecting significant CAD is probably related to the referral bias since all the patients in their cohort had known severe CAD with many patients having multivessel disease, and they all had previous revascularization of at least one vessel. Although it was a highly selective patient cohort, it is interesting to note that TID remains a robust marker for the presence and absence of severe CAD but also provided incremental value over myocardial perfusion markers alone. Therefore, the method used for the quantitative assessment and the definition of TID used in the study are of great importance if this criteria is applied on all patients having a MPI study. In their study, Gultekin et $\mathrm{al}^{22}$ have found that the optimal cut-off value for detecting significant obstructive CAD for the stress-rest $\mathrm{LV}$ volume ratio was 1.20 with the imaging protocol used in their patient population, for both exercise stress and vasodilator SPECT MPI. This finding raises the question of the reliability of the TID ratio measurement and its definition.

Different studies have shown that reported TID ratio or index can vary according to the radiotracer used, the MPI data acquisition protocol, the use of attenuation correction or not, the gender, the type of stress modality, and the threshold used to define TID. It is therefore mandatory to establish the normal values according to the above-mentioned variables in order to make TID a reproducible and useful index for CAD diagnosis and prognosis. The upper normal limit of TID for dual radioisotope exercise rest ${ }^{201}$ thallium/stress $99 \mathrm{mTc}$-labeled MPI radiotracer has been defined at 1.22 , while other upper normal limits for TID with rest ${ }^{201}$ thallium and same-day pharmacologic stress were 1.27 for dipyridamole, 1.40 for dobutamine, and 1.35 for adenosine. ${ }^{23} \mathrm{Xu}$ et $\mathrm{al}^{24}$ also reported the upper normal limit of TID using same-day rest-exercise stress $99 \mathrm{mTc}-$ sestamibi injection sequence. Their TID ratios were automatically derived using QPS/QGS software from static stress/rest MPI study (TID) and from gated stress/ rest MPI study from the end-diastolic phase $\left(\mathrm{TID}_{\mathrm{ed}}\right)$ and end-systolic phase $\left(\mathrm{TID}_{\mathrm{es}}\right)$. In a relatively large database obtained from low likelihood CAD patients, the upper limits were 1.19 for TID, 1.23 for $\mathrm{TID}_{\mathrm{ed}}$, and 1.46 for TID $_{\text {es. }}$ The use of TID ratios derived from the gated SPECT end-diastolic and end-systolic phases can be very useful in order to avoid averaging errors seen from the static MPI studies. Although previous studies have demonstrated that variations in gender or heart size may result in different TID thresholds (related to smaller absolute LV volumes in female patients), $\mathrm{Xu}$ et al ${ }^{24}$ did 
not find any statistically significant difference between TID ratios observed in patients with small hearts versus those with regular hearts.

As with any quantitative imaging parameter derived from radionuclide procedures, it is mandatory to obtain normal upper limit values of a given parameter derived from a large normal data base in order to define with more precision the standard deviation. Furthermore, all the technical and/or clinical variables which can potentially modify the normal values must be taken into consideration in establishing the clinical application of the given parameter. Numerous studies have now demonstrated the incremental diagnostic and prognostic values of the finding of TID during both treadmill exercise and vasodilator SPECT MPI. Measurement of TID should be an integral part of both SPECT MPI analysis and report on a routine basis with specification of the upper limit value used considering the above-mentioned criteria. The values used to define TID should be adapted to the specific imaging protocol and stress modality used in a given laboratory in order to obtain an optimal diagnostic accuracy.

\section{Disclosure}

The authors declare that they have no conflict of interest.

\section{References}

1. Iskandrian AS, Heo J, Nguyen T, Lyons E, Paugh E. Left ventricular dilatation and pulmonary uptake after single-photon emission computer tomography using thallium-201 during adenosine-induced coronary hyperemia. Am J Cardiol 1990;66:807-11.

2. McLaughlin MG, Danias PG. Transient ischemic dilation: A powerful diagnostic and prognostic finding of stress myocardial perfusion imaging. J Nucl Cardiol 2002;9:663-7.

3. Abidov A, Bax JJ, Hayes SW, Cohen I, Gerlach J, Kang X, et al. Transient ischemic dilation ratio of the left ventricle is a significant predictor of future cardiac events in patients with otherwise normal myocardial perfusion SPECT. J Am Coll Cardiol 2003;42:1818-25.

4. Mazzanti M, Germano G, Kiat H, Kavanagh PB, Alexanderson E, Friedman JD, et al. Identification of severe and extensive coronary artery disease by automatic measurement of transient ischemic dilation of the left ventricle in dual-isotope myocardial perfusion SPECT. J Am Coll Cardiol 1996;27:1612-20.

5. Abidov A, Germano G, Berman DS. Transient ischemic dilation ratio: A universal high-risk diagnostic marker in myocardial perfusion imaging. J Nucl Cardiol 2007;14:497-500.

6. Chouraki P, Rodrigues EA, Berman DS, Maddahi J. Significance of dipyridamole-induced transient dilation of the left ventricle during thallium-201 scintigraphy in suspected coronary artery disease. Am J Cardiol 1990;66:689-94.

7. Valdiviezo C, Motivala AA, Hachamovitch R, Chamarthy M, Navarro PC, Ostfeld RJ, et al. The significance of transient dilation in the setting of otherwise normal SPECT radionuclide myocardial perfusion images. J Nucl Cardiol 2011;18:220-9.

8. Takeishi Y, Tono-oka I, Ikeda K, Komatani A, Tsuiki K, Yasui S. Dilatation of the left ventricular cavity on dipyridamole thallium-
201 imaging: A new marker of triple-vessel disease. Am Heart J 1991;121:466-75.

9. McLellan JR, Travin MI, Herman SD, Baron JI, Golub RJ, Gallagher JJ, et al. Prognostic importance of scintigraphic left ventricular cavity dilation during intravenous dipyridamole technetium-99m sestamibi myocardial tomographic imaging in predicting coronary events. Am J Cardiol 1997;79:600-5.

10. Veilleux M, Lette J, Mansur A, Bertrand C, Cerino M, Picard M, et al. Prognostic implications of transient left ventricular cavity dilation during exercise and dipyridamole-thallium imaging. Can $\mathbf{J}$ Cardiol 1994;10:259-62.

11. Heiba SI, Cerqueira MD, Jacobson AF, Ferreira MJ. The value and correlates of left ventricular cavity assessment in dipyridamole ${ }^{201}$ T1 SPET studies. Nucl Med Commun 1998;19:443-9.

12. Marcassa C, Galli M, Baroffio C, Campini R, Giannuzzi P. Transient left ventricular dilation at quantitative stress-rest sestamibi tomography: Clinical, electrocardiographic, and angiographic correlates. J Nucl Cardiol 1999;6:397-405

13. Hansen CL, Sangrigoli R, Nkadi E, Kramer M. Comparison of pulmonary uptake with transient cavity dilation after exercise thallium201 perfusion imaging. J Am Coll Cardiol 1999;33:1323-7.

14. Hung GU, Lee KW, Chen CP, Lin WY, Yang KT. Relationship of transient ischemic dilation in dipyridamole myocardial perfusion imaging and stress-induced changes of functional parameters evaluated by Tl-201 gated SPECT. J Nucl Cardiol 2005;12:268-75.

15. Abidov A, Berman DS. Transient ischemic dilation associated with poststress myocardial stunning of the left ventricle in vasodilator stress myocardial perfusion SPECT: True marker of severe ischemia. J Nucl Cardiol 2005;12:258-60.

16. Weiss AT, Berman DS, Lew AS, Nielsen J, Potkin B, Swan HJ, et al. Transient ischemic dilation of the left ventricle on stress thallium-201 scintigraphy: A marker of severe and extensive coronary artery disease. J Am Coll Cardiol 1987;9:752-75.

17. Bestetti A, Di Leo C, Alessi A, Triulzi A, Tagliabue L, Tarolo GL. Post-stress end-systolic left ventricular dilation: A marker of endocardial post-ischemic stunning. Nucl Med Commun 2001;22:685-93.

18. Van Tosh A, Hecht S, Berger M, Roberti R, Luna E, Horowitz SF. Exercise echocardiographic correlates of transient dilatation of the left ventricular cavity on exercise thallium-201 SPECT imaging. Chest 1994;106:1725-9.

19. Weinmann P, Moretti JL. Effects of dipyridamole on left ventricular function. J Nucl Cardiol 2007;7:103-6.

20. Emmett L, Van Gaal WJ, Magee M, Bass S, Ali O, Freedman SB, et al. Prospective evaluation of the impact of diabetes and left ventricular hypertrophy on the relationship between ischemia and transient ischemic dilation of the left ventricle on single-day adenosine Tc-99m myocardial perfusion imaging. J Nucl Cardiol 2008;15:638-43.

21. Smelly MP, Virnich DE, Williams KA, Ward RP. A hypertensive response to exercise is associated with transient ischemic dilation on myocardial perfusion SPECT imaging. J Nucl Cardiol 2007; 14:537-43.

22. Gultekin SS, Sadic M, Bilgin M, Koca G, Acikel S, Yeter E, et al. The value of transient ischemic dilation for detecting restenosis after coronary artery revascularization. J Nucl Cardiol. 2016. doi: 10.1007/s12350-016-0607-z.

23. Katz JS, Ruisi M, Giedd KN, Rachko M. Assessment of transient ischemic dilation (TID) ratio in gated SPECT myocardial perfusion imaging (MPI) using regadenoson, a new agent for pharmacologic stress testing. J Nucl Cardiol 2012;19:727-34.

24. Xu Y, Arsanjani R, Clond M, Hyun M, Lemley M, Fish M, et al. Transient ischemic dilation for coronary artery disease in quantitative analysis of same-day sestamibi myocardial perfusion SPECT. J Nucl Cardiol 2012;19:465-73. 